\title{
WEB ENABLED BASED FACE RECOGNITION USING PARTITIONED ITERATED FUNCTION SYSTEM
}

\author{
Amol D.Potgantwar \\ Lecturer Computer Engg Dept \\ SITRC Nashik
}

\author{
Dr.S.G.Bhirud \\ Asst. Prof. Computer Engg Dept \\ VJTI Mumbai
}

\begin{abstract}
Many conventional methods of face recognition depend solely on appearance and model based. However there is inbuilt degree of self-similarity in the image of faces, which can be efficiently utilized through representation exploiting self-transformations, known as Iterated Function System (IFS).

Interestingly, virtually all images of natural or man-made objects, show region wise self similarity although they may not be globally self similar. Such objects can be represented by Partitioned Iterated Function System (PIFS) very compactly.

Hence, we propose is to carry out the face recognition using Partitioned Iterated Function System. This approach has been tested upon the 106 images of 27 persons using FERET database. The results obtained under the variance, rotation and scaling are outperforming. In this approach we carried out face recognition based on PIFS representation and matching carried out in the PIFS code domain, which is more efficient than correlation in the image domain.
\end{abstract}

The recognition method is efficient in terms of time complexity as the PIFS code of reference faces are built off-line and recognition of query object involves only comparison of its PIFS code with those in the database online.

\section{Keywords}

Alpha, Luminance, Fractals

\section{INTRODUCTION}

In recent years, face recognition has received more and more attention due to its benefit of being a passive, nonintrusive system to verify personal identity in a natural and friendly way. It has many potential application areas ranging from access control, mug shots searching, security monitoring, and surveillance systems. Face recognition is among the most challenging tasks in pattern recognition research due to its scientific challenges and potential applications.

There have been a lot of methods proposed for overcoming the difficulty of face recognition. Methods of face recognition can be divided into two approaches namely, feature geometry based and subspace analysis techniques. In feature geometry based approach, recognition is based on the relationship between human facial features such as eye(s), mouth, nose and face boundary. Subspace analysis approach attempts to capture and define the face as a whole. The face is treated as a two-dimensional pattern of intensity variation. The original image representation is highly redundant, and the dimensionality of this representation could be greatly reduced when only the face pattern is of interest.

Apart from these two approaches of face recognition In this paper, we consider the indexing problem for a class of images where it is possible to state fairly accurately the notion of a background and a foreground. Our experiments revolve around Given a library of reference images: I1, I2,..,In, and a query image Q, we want to preprocess the reference images to produce indices such that we can find the 'closest' image Ii to Q.Important subset of this class, namely, photographs of humans (such as those used in corporate identity cards, or those clicked by an automatic teller machine camera). Unlike images generated under structured lighting conditions (such as those of nuts and bolts in factory plants), faces with facial and tonsural hair growth have a predominant texture.

Traditional segmentation based techniques do not work well in such cases, and many interesting $[2,12]$ approaches fail. Fractals are important mathematical entities that have the ability to represent natural unstructured entities such as face, hair, and trees against the background in a photograph. Fractal descriptors are also compact, and therefore, have been used for compression. Indeed, the fractal subdivision method of chopping an image may be viewed as an automatic segmentation algorithm. The biggest impediment in using fractal descriptors for indexing is the one-tomany relationship between an image and fractal descriptors. Many descriptors can converge (using the fractal paradigm, made precise in Section 3) to the same image. In this paper, we study the use of fractal indices for general image indexing, and exemplify it with faces as the domain. Note that no assumption is made of "zeroing background" unlike approaches such as the venerable eigenfaces [11].

The rest of this paper is organized as follows. In the next section, we summarize previous work in this area. In Section 3 we provide the theoretical background for this work. In Section 4 our implementation is discussed along with sample results. Final remarks are made in Section 5

\section{RELATED WORK}

Although many researchers for the purpose of image compression have utilized the Iterated Function System (IFS) structure of fractal object representation, there have been very few attempts directed towards object indexing or recognition. In [4] the authors have presented a somewhat restricted recognition scheme applicable to the specific domain of L-System fractals and tested their technique on binary synthetic plant images generated by the L-System.

In [8] a recognition method is suggested which (i) works on 
binary images, and (ii) which is based on applying the reference set of Partitioned Iterated Function System (PIFS) codes on the query object and finding out the code which produces minimum change. The query is then recognized to be the object corresponding to that PIFS code, if the change found out is less than a threshold. This technique is not very interesting for the indexing problem because the comparison happens in the image domain, and not in the domain of indices.

In [7] the authors present a technique of indexing and contentbased retrieval by a set of PIFS fractal parameters without dealing with the theory of proximity of PIFS code.for visually similar The system proposed in [3] has the interesting property of being invariant under two classes of pixel intensity transformations: illumination or color alterations. The system can be used both by sketches, and the query by example paradigms. As seen in Section 4 and Figure 4, our system is more tolerant to semantic content changes.

In [13], a joint fractal coding technique is used for image retrieval, and compared with wavelet coding. They conclude that wavelet transform approach performs more effectively in contentbased similarity comparison on those images which contain strong texture features, whereas fractal coding approach performs relatively more uniformly well for various type of images. Unlike our experiments on faces, the conclusions are drawn on synthetic Broadatz texture images. The use of a joint fractal coding is different from our canonical coding.

\section{THEORETICAL BASIS}

In this section, we first introduce a few definitions, lemmas and theorems. The notion of complete and compact metric spaces, and the Hausdorff metric $h$ are as formulated in [1]. These are presented in the context of any set, and therefore are applicable to images when viewed as sets.

Definition 3.1 A transformation $w: \mathfrak{R}^{2} \rightarrow \mathfrak{R}^{2}$ of the form $\mathrm{w}(\mathrm{x}, \mathrm{y})=(\mathrm{ax}+\mathrm{by}+\mathrm{e}, \mathrm{cx}+\mathrm{dy}+\mathrm{f})$, where $\mathrm{a}, \mathrm{b}, \mathrm{c}, \mathrm{d}$, e and $\mathrm{f}$ are real numbers is called a 2-D affine linear transformation. The numbers $\mathrm{a}, \mathrm{b}, \mathrm{c}, \mathrm{d}, \mathrm{e}$, and $\mathrm{f}$ are called the parameters of the transformation.

Definition 3.2 A transformation $\mathrm{w}: \mathrm{X} \rightarrow \mathrm{X}$ on a metric space $(\mathrm{X}, \mathrm{d})$ is called contraction mapping if there is a constant $0 \leq \mathrm{s}<1$ such that $\mathrm{d}(\mathrm{w}(\mathrm{x}), \mathrm{w}(\mathrm{y})) \leq \mathrm{sd}(\mathrm{x}, \mathrm{y}), \forall \mathrm{x}$, $\mathrm{y} \in \mathrm{X}$. Any such number $\mathrm{s}$ is called a contractivity factor (CF) for $\mathrm{w}$.

Definition 3.3 An iterated function system (IFS) consists of a complete metric space $(X, d)$ together with a finite set of contractive mappings $w_{n}: \mathrm{X} \rightarrow \mathrm{X}$, with respective contractivity factors $\mathrm{sn}, \mathrm{n}=1,2,3, \ldots, \mathrm{N}$. The notation used for the IFS code is $\mathrm{W}=\left\{\mathrm{X} ; w_{n}, \mathrm{n}=1,2, \ldots, \mathrm{N}\right\}$ and its $\mathrm{CF}$ is $\mathrm{s}=\max$ $\left\{s_{n}: \mathrm{n}=1,2, \ldots, \mathrm{N}\right\}$. The set of parametric values for all the individual maps taken together is called the parameter of W.

Table 3.1 shows the IFS code for a 'fern' leaf using the notations of affine transformation as in Definition 3.1. IFS codes are compact and converge to an image (also known as a fixed point) from which they are produced (see Figure 3.1(a)).

Table 3.1 The IFS code for ferm.A few number generate a nice image as shown in fig 3.1nice image as shown in fig 3.1

\begin{tabular}{|c|c|c|c|c|c|}
\hline $\mathrm{a}$ & $\mathrm{b}$ & $\mathrm{c}$ & $\mathrm{d}$ & $\mathrm{e}$ & $\mathrm{f}$ \\
\hline 0.00 & 0.00 & 0.00 & 0.16 & 0.00 & 0.00 \\
0.85 & 0.04 & -0.04 & 0.85 & 0.00 & 1.60 \\
0.20 & -0.26 & 0.23 & 0.22 & 0.00 & 1.60 \\
-0.15 & 0.28 & 0.26 & 0.24 & 0.00 & 0.44 \\
\hline
\end{tabular}

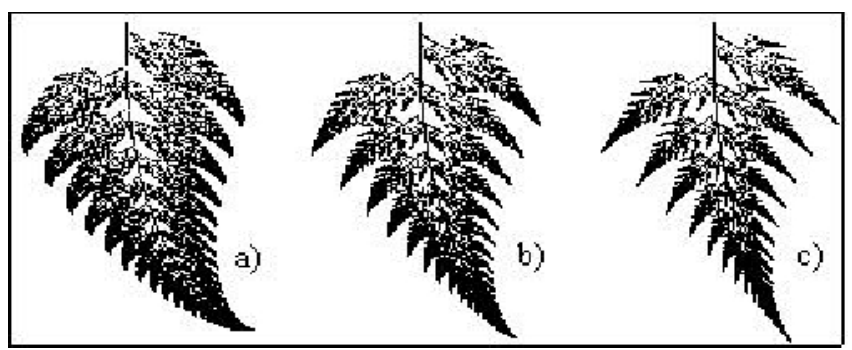

Figure 3.1 (a) An image obtained as the fixed point of the code in Table 3.1. (b) Another image obtained from the same code with the parameter a(1) changed to 0.80 . (c) Same as (b) with a (1) $=\mathbf{0 . 7 5}$.

IFS codes are also well behaved as illustrated in above Figure 3.1.This result may be stated formally as follows. Let $(\mathrm{P}, \mathrm{dp})$ and $(\mathrm{X}, \mathrm{d})$ be metric spaces. Let $\mathrm{w}: \mathrm{P} \in \mathrm{X} \rightarrow \mathrm{X}$ be a family of contraction mappings on $\mathrm{X}$ with contractivity factor $0 s<1$.That is, for each $\mathrm{p} \in \mathrm{P}, \mathrm{w}(\mathrm{p}$, .) is a contraction mapping on $X$. For each fixed $\mathrm{x} \in \mathrm{X}$, let $\mathrm{w}$ be continuous on $\mathrm{p}$.Then the fix ed point of $w$ depends continuously on $p$. That is $x: \mathrm{P} \rightarrow \mathrm{X}$ is continuous. Unfortunately,IFS codes are not unique as shown in Table 3.1
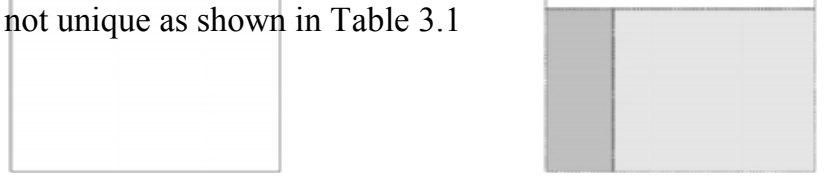

Figure 3.2 Two different contractive mapping may be obtained for a square, shown on the left. The first one is to use four squares each of one fourth area that tile the rectangle. The second mapping is to use three rectangles shown in the picture.

Whereas IFS deals with images that are self similar, the generalized notion of the Partitioned IFS is important for most images (anisotropic and non homogeneously scaled) that one deals with in real life. The key notion (formalized 
below) is to look at subsets of the image, rather than the entire image.

Definition 3.4 Let $(\mathrm{X}, \mathrm{d})$ be a compact metric space. Let $\mathrm{D}$ be a nonempty subset of $\mathrm{X}$. Let $\mathrm{w}: \mathrm{D} \rightarrow \mathrm{X}$ and let $\mathrm{s}$ be a real number with $0 \leq \mathrm{s}<1$. If $\mathrm{d}(\mathrm{w}(\mathrm{x}), \mathrm{w}(\mathrm{y})) \leq \mathrm{sd}(\mathrm{x}, \mathrm{y}), \forall$ $\mathrm{x}, \mathrm{y} \in \mathrm{D}$, then $\mathrm{w}$ is called a local contraction mapping on $(\mathrm{X}, \mathrm{d})$ with CF s. If there is a set of such w's written as $w_{i}$ with CFs $s_{i}$,for $\mathrm{i}=1,2, \ldots, \mathrm{N}$, then $\{$ wi : Di $\rightarrow \mathrm{X} ; \mathrm{i}=1,2$, ...,N $\}$ is called a partitioned iterated function system (PIFS). The number $\mathrm{s}=\max \left\{s_{i}: \mathrm{i}=1,2, \ldots, \mathrm{N}\right\}$ is the $\mathrm{CF}$ of the PIFS.

\subsection{Face Recognition Using PIFS}

All the above notions are self-contained and apply to any set. These definitions are mapped to gray level images (for example, see $[5,6])$. A PIFS $\{$ wi $\}$ for an image $\Psi$ is defined by wi $(x, y, \Psi)$ $=(\mathrm{wi}(\mathrm{x}, \mathrm{y}), \mathrm{vi}(\Psi))$, where $v i$ maps the intensity and is of the form $\operatorname{vi}(\mathrm{x})=S_{i} \mathrm{x}+O_{i} \cdot S_{i}$ performs a contrast scaling and $O_{i}$ a luminance shift.

Figure 3.3 shows how Range Block \& Domain Block are partitioned for calculating the contrast scaling \& luminance shift.Range Block are partitioned using $4 * 4$ nonoverlapping block \& Domain Block are partitioned using 64*64 overlapping block as shown in fig 3.3 \& calculate contrast scaling factor called alpha( $\alpha)$ and luminance $\operatorname{shift}(o)$ by using formula $1 \& 2$ respectively shown below.

$$
\begin{gathered}
\alpha=\frac{n \sum_{i=1}^{n} d_{i} r_{i}-\sum_{i=1}^{n} d_{i} \sum_{i=1}^{n} r_{i}}{n \sum_{i=1}^{n} d_{i}{ }^{2}-\left(\sum_{i=1}^{n} d_{i}\right)^{2}} \\
o=\frac{1}{n}\left(\sum_{i=1}^{n} r_{i}-\sum_{i=1}^{n} d_{i}\right)
\end{gathered}
$$

Definition 3.5 The parameters of $w_{i}$ along with the si and $O_{i}$ factors of vi underlying $w_{i}$ are called the parameters of $w_{i}$. The parameters of all the $w_{i}$ 's constituting a PIFS code are called the parameters of the code.

Definition 3.6 dw is a metric to compute the distance between two PIFS codes by summing up the term-wise absolute Text Box Copyright

differences between the parameters of the two codes. The entire theoretical framework discussed so far is needed for formally stating the following lemma which makes indexing by PIFS
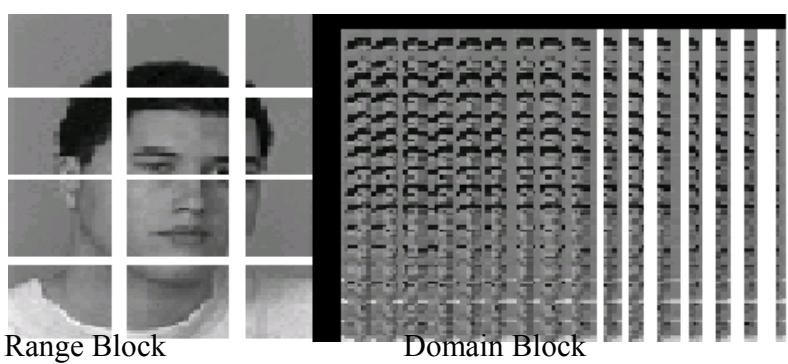

Lemma 3.1 Given two images I1 and I2 with distance d( I1, I2 ) under the metric $\mathrm{d}$, and the PIFS code $\mathbf{W} 1$ (with parameter p1) approximating I1 with error $\varepsilon 1$, there exists a PIFS W2 (with parameter p2) encoding I2 with error $\varepsilon 2$, such that

$\mathrm{dw}(\mathbf{W} 1, \mathbf{W} 2) \leq \mathrm{k} 1 \mathrm{~d}(\mathrm{I} 1, \mathrm{I} 2)$ (1) and

$$
\mathrm{d}(\mathrm{I} 1, \mathrm{I} 2) \rightarrow 0 \wedge \varepsilon 1 \Rightarrow 0 \wedge 2 \rightarrow 0 \wedge \mathrm{dw}(\mathbf{W} 1, \mathbf{W} 2) \rightarrow 0 .
$$

Where $\mathrm{k} 1$ is a constant

Lemma 3.2 establishes the continuity of the parameters of the PIFS codes over the encoded images, under a metric dw chosen to measure the distance in the PIFS code domain.

It is important to note that for a given image, it is possible to generate more than one PIFS code; but for the purpose of object recognition, it is enough if we obtain a canonical PIFS code in a manner that adheres to the proof. A collection of several canonical PIFS codes generated for a set of reference images constitute the database for carrying out object indexing.

\section{RESULT}

We have tested the PIFS based face recognition method on more than 30 human face images of 127 images in FERET database. In our system, one can present a query, by selecting randomly generated images from the FERET database file.Once a query face image is submitted it will retrieve face image from reference database image by using PIFS parameter \& display with mathching percentage.Our experimental result of a query image $\& 3$ reference images shows how a query image has been recognize by calculating the PIFS code \& matching with a minimum distance from reference image PIFS code. Addition of the absolute difference pixel wise between the domain block \& range block and that distance is called as minimum distance for that domain block with the range block. Figure 5 depicts the performance of our system quantitatively. This data shows that our system satisfies the level of accuracy on mugshotlike images as suggested in [8]. 


\begin{tabular}{|l|l|l|}
\hline 15 & 0.0000 & 255.0000 \\
\hline 16 & 0.0000 & 255.0000 \\
\hline
\end{tabular}

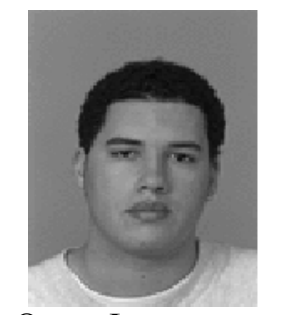

Query Image
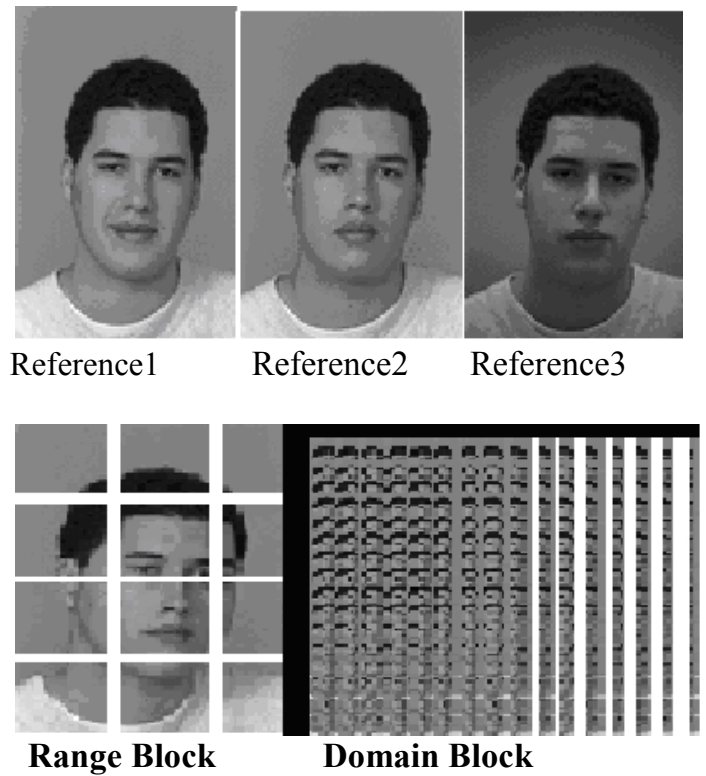

Table 4.1 PIFS Code for Query Image

\begin{tabular}{|c|c|c|}
\hline Sr.No. & Alpha & Luminance \\
\hline 1 & -0.0704 & 80.0560 \\
\hline 2 & 0.2958 & 53.3265 \\
\hline 3 & -0.0551 & 96.6948 \\
\hline 4 & -0.1076 & 107.0728 \\
\hline 5 & -0.0767 & 69.0321 \\
\hline 6 & -0.1511 & 80.3522 \\
\hline 7 & -0.0243 & 82.2535 \\
\hline 8 & 0.2157 & 52.1793 \\
\hline 9 & 0.9736 & 10.4714 \\
\hline 10 & 1.0122 & 1.4941 \\
\hline 11 & 0.8659 & 36.3919 \\
\hline 12 & 0.7436 & 65.4560 \\
\hline 13 & 0.0000 & 255.0000 \\
\hline 14 & 0.0000 & 255.0000 \\
\hline
\end{tabular}

Table 4.2 Code of Best Match Image for Reference1 Image

\begin{tabular}{|c|c|c|c|}
\hline $\begin{array}{l}\text { Sr. } \\
\text { No. }\end{array}$ & Min.Distance & Alfa & Luminance \\
\hline 1 & 2.8365 & -0.0704 & 80.0560 \\
\hline 2 & 0.9201 & 0.2958 & 53.3265 \\
\hline 3 & 14.6547 & 0.0551 & 96.6948 \\
\hline 4 & 0.9723 & -0.1076 & 107.0728 \\
\hline 5 & 32.3073 & -0.0767 & 69.0321 \\
\hline 6 & 5.3129 & -0.1511 & 80.3522 \\
\hline 7 & 0.0680 & -0.0243 & 82.2535 \\
\hline 8 & 8.2117 & 0.2157 & 52.1793 \\
\hline 9 & 3.7339 & 0.9736 & 10.4714 \\
\hline 10 & 4.6050 & 1.0122 & 1.4941 \\
\hline 11 & 2.2078 & 0.8659 & 36.3919 \\
\hline 12 & 0.0642 & 0.7436 & 65.4560 \\
\hline 13 & 0.0000 & 0.0000 & 255.0000 \\
\hline 14 & 0.0000 & 0.0000 & 255.0000 \\
\hline 15 & 0.0000 & 0.0000 & 255.0000 \\
\hline 16 & 0.0000 & 0.0000 & 255.0000 \\
\hline
\end{tabular}

Table 4.3 Code of Best Match Image for Reference2 
Image

\begin{tabular}{|c|c|c|c|}
\hline $\begin{array}{l}\text { Sr. } \\
\text { No. }\end{array}$ & Min.Distance & Alfa & Luminance \\
\hline 1 & 58.8104 & -0.2760 & 5.3563 \\
\hline 2 & 1.3773 & 0.6118 & 12.8748 \\
\hline 3 & 5.4335 & -0.0634 & 135.4312 \\
\hline 4 & 0.1164 & 0.6303 & 82.5332 \\
\hline 5 & 32.3073 & -0.7734 & 223.0706 \\
\hline 6 & 0.0325 & -0.2107 & 127.1383 \\
\hline 7 & 1.5758 & -0.1852 & 146.1065 \\
\hline 8 & 11.2975 & 0.6441 & 40.8512 \\
\hline 9 & 0.0369 & 0.8866 & 29.3168 \\
\hline 10 & 0.8457 & 0.8015 & -12.2366 \\
\hline 11 & 0.1581 & 0.9096 & 22.7633 \\
\hline 12 & 0.8378 & 0.5793 & 108.2082 \\
\hline 13 & 0.0000 & 0.0000 & 255.0000 \\
\hline 14 & 0.0000 & 0.0000 & 255.0000 \\
\hline 15 & 0.0000 & 0.0000 & 255.0000 \\
\hline 16 & 0.0000 & 0.0000 & 255.0000 \\
\hline
\end{tabular}

Table 4.4 Code of Best Match Image for Reference3 Image

\begin{tabular}{|l|l|l|l|}
\hline $\begin{array}{l}\text { Sr. } \\
\text { No. }\end{array}$ & Min.Distance & Alfa & Luminace \\
\hline
\end{tabular}

\begin{tabular}{|c|c|c|c|}
\hline 1 & 77.3346 & -0.1293 & 147.0552 \\
\hline 2 & 2.9309 & 0.5399 & 45.4376 \\
\hline 3 & 10.0825 & -0.0510 & 135.4312 \\
\hline 4 & 0.2928 & 0.6768 & 74.0749 \\
\hline 5 & 8.5696 & -0.7734 & 206.7640 \\
\hline 6 & 0.4224 & -0.1458 & 119.1349 \\
\hline 7 & 0.0551 & -0.2035 & 155.4577 \\
\hline 8 & 6.0648 & 0.8367 & 18.5146 \\
\hline 9 & 0.4011 & 0.6612 & 86.3518 \\
\hline 10 & 0.6536 & 1.0447 & -12.2366 \\
\hline 11 & 0.2760 & 0.7265 & 69.8904 \\
\hline 12 & 0.9409 & 0.5293 & 120.8622 \\
\hline 13 & 0.0000 & 0.0000 & 255.0000 \\
\hline 14 & 0.0000 & 0.0000 & 255.0000 \\
\hline 15 & 0.0000 & 0.0000 & 255.0000 \\
\hline 16 & 0.0000 & 0.0000 & 255.0000 \\
\hline
\end{tabular}

\section{CONCLUSION}

We have carried out the face recognition on the gray level images by PIFS code. Since the parameters of the PIFS code of reference database image and Query Image matches, we can recognize the query image online.

It is important to note that the PIFS code for an image, and the image itself do not have one to one correspondence. It is enough to be able to extract a canonical PIFS for a given image. This method can index objects, which are unstructured in general. For faces, this means that we do not need to know the background unlike the eigenfaces approach which uses, for example in FERET database, the location of eyes. This approach of recognition is less computation intensive as compared to many other methods like PCA based, LFA, HMM, etc 
[11] J. Wang, J. Li, and G. Wiederhold. "Simplicity: Semantics sensitive integrated matching for picture libraries". IEEE Transactions on Pattern Analysis and Machine Intelligence, 23:947-963, 2001.

[12] A.Zhang, B. Cheng, R.Acharya, and R. Menon. "Comparison of wavelet transforms and fractal coding in texture-based image retrieval ". In G.G. Grinstein and R.F. Erbacher, editors, Visual Data Exploration and Analysis III, volume 2656of SPIE Proceedings, pages 116-125, 1996.

[13] J.Wayman, A.Jain, D.Maltoni, D.Mario, “ Handbook of Biometric Systems", Springer Publication.

[14] S.Z.Li, A.K.Jain, "Handbook of Face Recognition", Springer Publication.

[15] Recent Advances In Face Recognition kresimir Delac,Mislav Grgic and Marrian Stewart Bartlett November 2008.

Figure 5. The error in our system. A number close to zero is good.

\section{REFERENCES}

[1] M. Barnsley, "Fractals Everywhere", Academic Press Professional, 1993.

[2] C. Carson, M. Thomas, S. Belongie, J. Hellerstein, and 豞, Malik., " Blobworld: a system for tregion-based image indexing and retrieval ", In Third International Conference on Visual Information Systems, pages 509-516, 1999.

[3] D. Holliday and A. Samal, "object recognition using L system fractals", Pattern Recognition Letters, 16 , 3342, 1995.

[4] A.Jacquin, "Image códinge. based on fractál theory of iterated contractive image transformations" IEEE Transactions on Image Processing, 1:18-30, 1992.

[5] S. Chandran and S. Kar, "Retrieving faces by PIFS Fractal Code", IEEE workshop on Applications of Computer Vision, pages 8-12,2002

[6] J. Marie-Julie and H. Essafi. Digital image indexing and retrieval by content using the Fractal transform for multimedia databases". In Proceedings of the 4th International Forum on Research and Technology Advances in Digital Libraries, pages 509-516, 1997.

[7] G. Neil and K. Curtis. "Shape recognition using fractal geometry". Pattern Recognition, 30:1957- 1969, 1997.

[8] A.Pentland and T. Choudhury. "Face recognition for smart environments". IEEE Computer, pages 50- 55, 2000.

[9] P. J. Phillips, H. Moon, S. A. Rizvi, and P. J. Rauss. "The FERET evaluation methodology for face-recognition algorithms". IEEE Transactions on Pattern Analysis and Machine Intelligence, 22(10): 1090-1104, 2000.

[10] M. Turk and A. Pentland. Eigenfaces for recognition. “Journal of Cognitive Neurosciences", pages 71-86, 1991. 